Molecular dynamics in tilted bilayer smectic phases: A proton nuclear magnetic resonance relaxometry study

A. Carvalho, P. J. Sebastião, A. C. Ribeiro, H. T. Nguyen, and M. Vilfan

Citation: The Journal of Chemical Physics 115, 10484 (2001); doi: 10.1063/1.1413744

View online: https://doi.org/10.1063/1.1413744

View Table of Contents: http://aip.scitation.org/toc/jcp/115/22

Published by the American Institute of Physics 


\title{
Molecular dynamics in tilted bilayer smectic phases: A proton nuclear magnetic resonance relaxometry study
}

\author{
A. Carvalho \\ Centro de Física da Matéria Condensada, Av. Prof. Gama Pinto 2, 1649-003 Lisboa, Portugal and Instituto \\ Superior Técnico, Av. Rovisco Pais, 1049-001 Lisboa, Portugal, and Escola Superior de Tecnologia da \\ Saúde de Lisboa, R. José Carlos dos Santos, 7, 1700-256 Lisboa, Portugal \\ P. J. Sebastião a) and A. C. Ribeiro \\ Centro de Física da Matéria Condensada, Av. Prof. Gama Pinto 2, 1649-003 Lisboa, Portugal and Instituto \\ Superior Técnico, Av. Rovisco Pais, 1049-001 Lisboa, Portugal \\ H. T. Nguyen \\ CNRS, Centre de Recherche Paul Pascal, Av. A. Schweitzer, F-33600 Pessac, France \\ M. Vilfan \\ Jozef Stefan Institute, Jamova 39, 1000 Ljubljana, Slovenia
}

(Received 25 April 2001; accepted 5 September 2001)

\begin{abstract}
A proton nuclear magnetic resonance (NMR) relaxation study of molecular dynamics in the liquid crystal 4-octylphenyl 2-chloro-4-(4-cyanobenzoyloxy)benzoate $\left(\mathrm{DB}_{8} \mathrm{Cl}\right)$ is presented. $\mathrm{DB}_{8} \mathrm{Cl}$ molecules possess a strong polar terminal group and form, in addition to the nematic phase, three different smectic phases: bilayer smectic $\mathrm{A}$, bilayer smectic $\mathrm{C}$, and anticliniclike smectic $\mathrm{C}$ phase. The proton spin-lattice relaxation times were measured in all mesophases over a broad frequency range of six decades by applying conventional and fast field-cycling NMR techniques. The parameters obtained in the analysis of the experimental data give quantitative information on molecular motions, particularly for the tilted smectic phases of $\mathrm{DB}_{8} \mathrm{Cl}$. In contrast to former conjectures, we found that the low-frequency relaxation in the bilayer smectic $\mathrm{C}$ phases results from director fluctuations about the layer normal, which occur without distortion of the layers, and from layer undulations, similar to those in the smectic A phase. In the low-temperature bilayer smectic C phase, a considerable slowing-down of molecular translational diffusion is observed. It confirms indirectly the anticlinic character of this mesophase. Measurements of angular dependence of the relaxation times at $60 \mathrm{MHz}$ support the conclusions obtained from the frequency dispersion data. (C) 2001 American Institute of Physics. [DOI: 10.1063/1.1413744]
\end{abstract}

\section{INTRODUCTION}

It is well-known that liquid crystals with strong polar terminal groups often present a great variety of mesophases, including the nematic phase and smectic $\mathrm{A}$ and $\mathrm{C}$ phases, consisting of monolayers, bilayers or partial-bilayers. ${ }^{1-6}$ The structures of these phases and transitions among them have been an object of detailed study by $\mathrm{x}$-ray diffraction, ${ }^{7-11}$ calorimetry, ${ }^{12-15}$ and nuclear magnetic resonance (NMR) ${ }^{16-23}$ Dielectric measurements and NMR relaxometry gave information on molecular dynamics, predominantly in the nematic and bilayer smectic A phases. ${ }^{16-22,24}$ On the other hand, the structural properties and dynamics in tilted smectic phases have been much less investigated and are still not well understood. In this paper we apply proton NMR relaxometry in a broad frequency range to study the peculiar molecular arrangement and mobility of such phases. We focus on the uniformly tilted bilayer smectic $\mathrm{C}$ phase $\left(\mathrm{SmC}_{2}\right)$, and on the anticliniclike phase $\left(\mathrm{SmC}_{\text {? }}\right)$, which appears at lower temperature, and which is-as far its proposed structure is concerned-between the alternating and the bilayer tilted phases. ${ }^{24,25}$

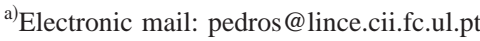

In NMR relaxometry of liquid crystals we are dealing with several mechanisms, i.e., types of molecular motion, responsible for the relaxation of nuclear spins. As different motional processes occur over different time scales they are usually assumed to be statistically independent and the coupling between them is neglected. Previous studies of molecular dynamics in compounds with polar end-groups have revealed that the relaxation mechanisms in the smectic A phase are not considerably different from those observed in the nematic phase. ${ }^{18,19,26-29}$ If protons are used as the molecular probe, the relaxation in the $\mathrm{MHz}$ regime of both mesophases is governed by reorientations of the whole molecule, fast conformational changes within the molecule, and translational self-diffusion. The latter affects only intermolecular proton interactions.

The most significant difference between a liquid crystal and isotropic fluids is observed in the $\mathrm{kHz}$ frequency range. ${ }^{30,31}$ Collective molecular reorientations, which are known as director fluctuations (DF), produce here a peculiar dependence of the spin-lattice relaxation rate $T_{1}^{-1}$ on the Larmor frequency $\nu$. In the nematic phase, $T_{1}^{-1}$ is proportional to $\nu^{-1 / 2}$ over several frequency decades as theoretically predicted by Pincus and experimentally definitely con- 


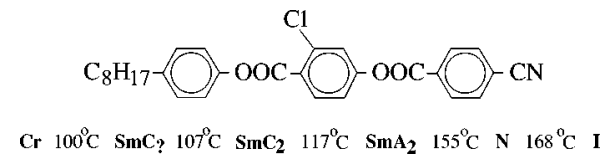

FIG. 1. Chemical structure and polymorphism of the $\mathrm{DB}_{8} \mathrm{Cl}$ compound.

firmed by Wölfel, Noack, and Stohrer. ${ }^{30,32}$ In the smectic A phase, collective orientational fluctuations are basically layer undulations. They seem to be less effective than nematic fluctuations as the spin-lattice relaxation time in the smectic A phase exceeds that of the nematic phase almost by one order of magnitude in the $\mathrm{kHz}$ regime. The relaxation rate associated with pure layer undulations should go as $T_{1}^{-1}$ $\propto \nu^{-1}$ due to the two-dimensional character of such fluctuations. ${ }^{26}$

The relaxation in the tilted smectic phases, composed of nonchiral molecules, has been up to now only marginally included in the papers describing the relaxation in liquid crystals. ${ }^{27,33,34}$ No definite conclusion on the frequency dependence of $T_{1}^{-1}$ in the $\mathrm{kHz}$ regime has been given. In some cases it was ascribed to nematiclike director fluctuations and in others to undulations as in the smectic A phase. This should be the reason for the comment of Vold and Vold that director fluctuations should be operative in tilted smectic C phase, ${ }^{35}$ but adequate theory and experiment are lacking. The situation has not much improved up to now. Recently, Acosta and Pusiol pointed out that both types of dispersion, $T_{1}^{-1}$ $\propto \nu^{-1 / 2}$ and $T_{1}^{-1} \propto \nu^{-1}$, are observed close to the smectic C-nematic phase transition. ${ }^{21}$ Their observation was only qualitative and assigned to the pre-transitional effects, i.e., pseudo nematic domains within the smectic phase. In order to throw additional light on this problem and on the molecular dynamics in tilted smectic phases generally, we decided to perform a comprehensive study of the NMR proton spinlattice relaxation in the liquid crystal 4-octylphenyl 2-chloro4-(4-cyanobenzoyloxy)benzoate, $\mathrm{DB}_{8} \mathrm{Cl}$ for short.

The $\mathrm{DB}_{8} \mathrm{Cl}$ compound shows four mesophases upon decreasing temperature: One nematic and three bilayer smectic phases (Fig. 1). ${ }^{9,15}$ The structures of the $\mathrm{SmA}_{2}$ and $\mathrm{SmC}_{2}$ phases are well understood. In the smectic $\mathrm{A}_{2}$ phase molecules are oriented perpendicular to the plane of the layer which has a thickness of approximately two molecular lengths as illustrated in Fig. 2. In the $\mathrm{SmC}_{2}$ phase, the molecular pairs are tilted with respect to the layer normal. The tilt angle $\theta$ depends on the temperature and amounts up to $30^{\circ}$ just above $107^{\circ} \mathrm{C}$. At this temperature the transition into another tilted smectic phase, which is denoted by $\mathrm{SmC}_{\text {? }}$,
$\mathrm{SmA}_{2}$

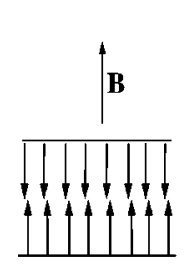

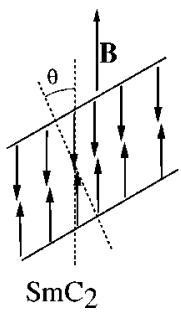

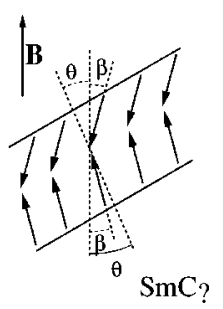

FIG. 2. Schematic representation of the structures of the $\mathrm{SmA}_{2}, \mathrm{SmC}_{2}$, and $\mathrm{SmC}_{\text {? }}$ phases of $\mathrm{DB}_{8} \mathrm{Cl}$. B represents the external magnetic field. takes place. On the basis of dielectric measurements, $\mathrm{x}$-rays diffraction and proton NMR studies an anticlinic type of ordering was proposed for this mesophase..$^{9,23,24,36}$ Within this model the anticlinic arrangement is superimposed on the "regular tilt." Within a bilayer, one layer of molecules are tilted for an angle $\theta+\beta$ and the other for the angle $\theta-\beta$ away from the layer normal (Fig. 2). $\beta$ was found to be about $13^{\circ} \pm 2^{\circ} .^{23}$

In this work we present a study of relaxation in all mesophases of $\mathrm{DB}_{8} \mathrm{Cl}$ in order to correlate the parameters obtained for the description of molecular dynamics in the $\mathrm{SmC}_{2}$ and $\mathrm{SmC}_{\text {? }}$ phases with those of the $\mathrm{N}$ and $\mathrm{SmA}_{2}$ phases. In Sec. II experimental procedure and results are described. In Sec. III we present the relaxation mechanisms used in the analysis of the measured relaxation times. The values of the parameters obtained in the fitting procedure are discussed in Sec. IV. They show significant differences between different mesophases and provide an insight into molecular dynamics, particularly for the tilted smectic phases. The conclusions are outlined in Sec. V.

\section{EXPERIMENTAL TECHNIQUES AND RESULTS}

The proton spin-lattice relaxation time $T_{1}$ of $\mathrm{DB}_{8} \mathrm{Cl}$ was measured as a function of Larmor frequency $(\nu)$, sample's orientation angle $(\Delta)$ and temperature $(T)$. In order to cover a broad Larmor frequency range from $\sim 500 \mathrm{~Hz}$ to $300 \mathrm{MHz}$, three different NMR spectrometers were used: a Bruker MSL 300 operating at $300 \mathrm{MHz}$; a Bruker SXP-4/100 for frequencies between 5 and $100 \mathrm{MHz}$, and a home-built fast fieldcycling spectrometer in the low-frequency regime. ${ }^{37}$ Different pulse sequences were applied according to different frequency ranges. The usual inversion-recovery pulse sequence was used to perform the $T_{1}$ measurements in the $\mathrm{MHz}$ frequency range. On the fast field-cycling spectrometer the sequence $\left(B_{H \rightarrow L}-\tau_{i}-B_{L \rightarrow H}-(\pi / 2)-F I D\right)$ was used, where $\tau_{i}$ is the evolution time, $(\pi / 2)$ is the radio frequency (rf) detecting pulse, and $B_{H \rightarrow L}, B_{L \rightarrow H}$ are transitions between the high and low magnetic fields, respectively. ${ }^{31}$ The measurements of the angular dependence of $T_{1}$ were performed at a frequency of $60 \mathrm{MHz}$ on the Bruker SXP spectrometer by using a step motor to achieve the angular resolution of $0.6^{\circ}$.

The $\mathrm{DB}_{8} \mathrm{Cl}$ sample was sealed in NMR glass tubes of $0.5 \mathrm{~mm}$ diameter under moderate vacuum $(<0.133 \mathrm{~Pa})$. Since the observed $T_{1}$ might depend somewhat on the procedure by which the mesophases were oriented in the magnetic field, we took care to perform all measurements after cooling the sample, in the presence of the external magnetic field, from the isotropic phase to the desired temperature at the rate $1{ }^{\circ} \mathrm{C} / \mathrm{min}$. In this way the nematic and smectic A phases are homogeneously oriented with the director parallel to the magnetic field everywhere in the sample. The normal to the layers in the smectic $\mathrm{A}_{2}$ phase has the same direction. When the sample is cooled into the smectic $\mathrm{C}_{2}$ phase, the molecules are still aligned along the magnetic field. ${ }^{38}$ This fact implies, however, an inclination of the smectic planes and consequently a decomposition of the uniform structure into domains. Each domain is characterized by the orientation of the layers' normal, i.e., by its polar and azimuthal 


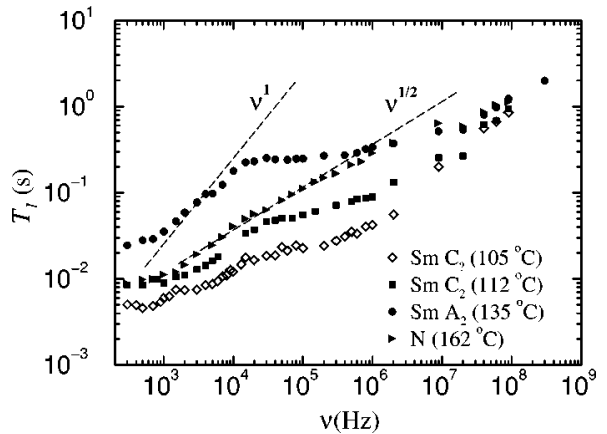

FIG. 3. Frequency dependence of the proton spin-lattice relaxation time $T_{1}(\nu)$ in the $\mathrm{N}, \mathrm{SmA}_{2}, \mathrm{SmC}_{2}$, and $\mathrm{SmC}_{\text {? }}$ phases of $\mathrm{DB}_{8} \mathrm{Cl}$. The dashed lines are guide lines to the characteristic slopes of the DF mechanism $\left(T_{1} \sim \nu^{1 / 2}\right.$ and $T_{1} \sim \nu^{1}$ ).

angles with respect to the magnetic field. Whereas the polar angle equals $\theta$ in all domains, the azimuthal angle assumes all values between 0 and $2 \pi$, with equal probability.

In Fig. 3 we present the experimental results of $T_{1}(\nu)$ at one temperature for each mesophase of $\mathrm{DB}_{8} \mathrm{Cl}$. The random error limits are estimated to be $\pm 10 \%$; for the sake of clarity the experimental error bars are omitted. The $T_{1}$ frequency dispersions in the $\mathrm{N}$ and $\mathrm{SmA}_{2}$ phases of $\mathrm{DB}_{8} \mathrm{Cl}$ are similar to those found in other liquid crystals. ${ }^{18,19,39}$ In both mesophases $T_{1}$ increases from the low to high frequencies but at intermediate frequencies there is a plateau in $T_{1}(\nu)$ in the $\mathrm{SmA}_{2}$ phase which is not observed in the $\mathrm{N}$ phase. In the $\mathrm{SmA}_{2}$ phase the dispersion $T_{1} \propto \nu^{1}$ is observed in one decade $\left(10^{3}-10^{4} \mathrm{~Hz}\right)$, whereas in the nematic phase the director fluctuations $\left(T_{1} \propto \nu^{1 / 2}\right)$ obviously govern the relaxation from $10^{3}$ to $10^{6} \mathrm{~Hz}$. In this frequency range $T_{1}$ in the nematic phase is considerably shorter than in the $\mathrm{SmA}_{2}$ phase.

A first inspection of the $T_{1}$ dispersion in the $\mathrm{SmC}_{2}$ phase (Fig. 3) shows partly the characteristics of the nematic and partly of the $\mathrm{SmA}_{2}$ phase, namely: (i) at low frequencies $\left(10^{3} \mathrm{~Hz}-4 \times 10^{4} \mathrm{~Hz}\right) T_{1}$ increases with frequency as in the $\mathrm{N}$ phase $\left(T_{1} \propto \nu^{1 / 2}\right)$; (ii) at intermediate frequencies $\left(4 \times 10^{3}\right.$ $\left.\mathrm{Hz}-10^{6} \mathrm{~Hz}\right) T_{1}(\nu)$ evidences a leveling-off which resembles the plateau in the $\mathrm{SmA}_{2}$ phase. In the $\mathrm{SmC}_{\text {? }}$ phase the spinlattice relaxation time is considerably shorter than in other mesophases over the whole frequency range studied. The shape of the dispersion is similar to the one observed for the $\mathrm{SmC}_{2}$ phase.

In Fig. 4 the angular dependences of the relaxation rate

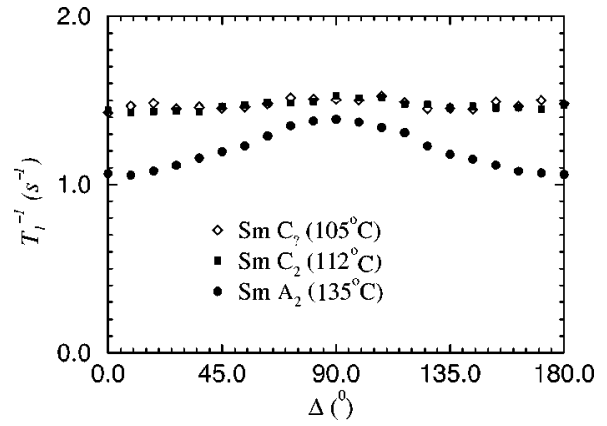

FIG. 4. Angular dependence of the proton spin-lattice relaxation rate $T_{1}^{-1}$ in the smectic phases of $\mathrm{DB}_{8} \mathrm{Cl}$ at $\nu=60 \mathrm{MHz}$.
$\left(T_{1}^{-1}(\Delta)\right)$ are presented for one temperature in each smectic mesophase at the frequency $60 \mathrm{MHz}$; for the sake of clarity the experimental error bars are omitted. The angular dependence is obtained by rotating the sample about an axis perpendicular to the external magnetic field. $\Delta$ denotes the angle of rotation of the sample with $\Delta=0$ denoting the position at which the cooling from the isotropic phase was performed. In the case of the smectic $A_{2}$ phase, the molecular director turned away from the magnetic field by sample rotation, does not realign for quite a long time. This enables the measurements of the spin relaxation rate with the director turned away by $\Delta$ from the direction of the magnetic field. Figure 4 shows that $T_{1}^{-1}(\Delta)$ exhibits a characteristic maximum at $90^{\circ}$ in the $\mathrm{SmA}_{2}$ phase. Rotating the tilted smectic phases, $\mathrm{SmC}_{2}$ and $\mathrm{SmC}_{?}$, results in the corresponding rotation of the layer planes. However, the molecules in each domain adjust their orientation closer to the magnetic field while preserving their tilt angle and the orientation of the layers. The director, which represents also in the tilted smectic phases the preferred direction of long axis, has therefore different orientations in different domains for $\Delta \neq 0 .{ }^{23}$ The angle of the director in each $m$ domain with respect to the magnetic field is denoted by $\delta_{m}$ and depends, naturally, on the angle $\Delta$ of the rotation of the sample. In both tilted smectic phases, $\mathrm{SmC}_{2}$ and $\mathrm{SmC}_{\text {, }}$, the NMR spectra show a well expressed angular dependence. ${ }^{23}$ On the other hand, the spin-lattice relaxation rates are almost independent of the sample orientation as shown in Fig. 4.

\section{RELAXATION MECHANISMS}

The proton spin-lattice relaxation measurements are quantitatively interpreted by a global target nonlinear leastsquare fitting minimization procedure in which a linear combination of relaxation mechanisms' models is used. The most important relaxation mechanisms are:

(1) Translational self-diffusion (SD), modulating intermolecular magnetic dipolar proton interactions;

(2) director fluctuations (DF), expected to be effective at low frequencies;

(3) molecular rotations/reorientations (R), acting on the intra-molecular interactions; i.e., magnetic dipolar interactions among protons in the same molecule.

Assuming that these relaxation mechanisms are statistically independent, the measured relaxation rate can be interpreted as a sum of three contributions:

$$
\frac{1}{T_{1}}=\left(\frac{1}{T_{1}}\right)_{\mathrm{SD}}+\left(\frac{1}{T_{1}}\right)_{\mathrm{DF}}+\left(\frac{1}{T_{1}}\right)_{\mathrm{R}} .
$$

The following relaxation models were used in the fitting procedure of the theory to the experimental data:

(1) Anisotropic translational self-diffusion as described by Vilfan and Žumer for nematic and smectic A phases. ${ }^{40,41}$ Its contribution to the relaxation rate induced by the time-modulation of intermolecular interactions is 


$$
\begin{aligned}
\left(\frac{1}{T_{1}}\right)_{\mathrm{SD}}= & \frac{9}{8}\left(\frac{\mu_{0}}{4 \pi}\right)^{2} \gamma^{4} \hbar^{2} \frac{n \tau_{D \perp}}{d^{3}} \\
& \times Q\left(\nu \tau_{D \perp}, \frac{\left\langle r_{\perp}^{2}\right\rangle}{d^{2}}, \frac{D_{\|}}{D_{\perp}}, \frac{l}{d}, \delta\right),
\end{aligned}
$$

where $\gamma$ is the proton gyromagnetic ratio, $n$ is the number of spins per unit volume, $d$ the distance of closest approach of two proton spins on neighboring molecules, $\left\langle r_{\perp}^{2}\right\rangle$ is the mean square jump distance, and $\tau_{D \perp}$ the average time between two successive jumps in the plane of the layer for the smectic phases and in the direction perpendicular to the director for the nematic phase. Similarly, $\ell$ denotes layers' spacing in the smectic phases and molecular length in the nematic phase. The relaxation rate $\left(T_{1}^{-1}\right)_{\mathrm{SD}}$ depends further on the ratio $D_{\|} /$ $D_{\perp}$ of the diffusion coefficients in two perpendicular directions and on the angle $\delta$ between the molecular director and the magnetic field. The dimensionless function $Q$, which determines the frequency dependence of $\left(T_{1}^{-1}\right)_{\mathrm{SD}}$, depends on the details of the diffusion process and on the spatial molecular arrangement. It is different for the nematic and for the smectic A phase and was evaluated numerically for both cases in Refs. 40 and 41. It should be also mentioned that the parameters $D_{\|}$and $D_{\perp}$ are related to the ones referred for the perfectly ordered nematic and smectic A structures $\left(D_{\|}^{0}\right.$ and $D_{\perp}^{0}$ ) through the orientational order parameter $S^{42}$

$$
\begin{aligned}
& D_{\|}=\frac{2 D_{\perp}^{0}+D_{\|}^{0}}{3}(1-S)+S D_{\|}^{0}, \\
& D_{\perp}=\frac{2 D_{\perp}^{0}+D_{\|}^{0}}{3}(1-S)+S D_{\perp}^{0} .
\end{aligned}
$$

As there is no specific model for the relaxation induced by translational self-diffusion in the tilted smectic phases, the above model for the SmA phase was used also in the analysis of the $\mathrm{SmC}_{2}$ and $\mathrm{SmC}_{\text {? }}$ phases. The approximation is reasonable in view of a weak dependence of $\left(T_{1}^{-1}\right)_{\mathrm{SD}}$ on the angle between the molecular director and the external magnetic field.

In fitting the contribution of translational self-diffusion, $\left(T_{1}^{-1}\right)_{\mathrm{SD}}$, to the experimental data, $\tau_{D \perp}$ was the only free parameter. The values of other parameters used in the fit are: $n \simeq 3.4 \times 10^{28}$ spins $\mathrm{m}^{-3}$ (calculated for $\mathrm{DB}_{8} \mathrm{Cl}$ from a specific density of $\sim 1 \mathrm{~g} / \mathrm{cm}^{3}$ and taking into account the number of proton spins present in each molecule), $\left\langle r_{\perp}^{2}\right\rangle / d^{2}=1$; $d \simeq 5.0 \times 10^{-10} \mathrm{~m}$ in the nematic and $\mathrm{SmA}_{2}$ phases, and $d$ $\simeq 4.8 \times 10^{-10} \mathrm{~m}$ in the $\mathrm{SmC}$ phases (in agreement with the decreasing of molecular area with temperature) $;{ }^{43} l \simeq 28$ $\times 10^{-10} \mathrm{~m}$ (estimated from the $\mathrm{DB}_{8} \mathrm{Cl}$ molecule in its stretched conformation). As for $D_{\|} / D_{\perp}$, the values obtained in the literature for similar compounds were used, i.e., $D_{\|} / D_{\perp}$ is 1.4 in the nematic phase, 1 in the smectic $\mathrm{A}_{2}$ and $\mathrm{C}_{2}$ phases and 0.18 in the smectic $\mathrm{C}_{\text {? }}$ phase. ${ }^{40,41,44}$ Anyway, the value of this parameter, taken within reasonable limits does not affect appreciably the fit;

(2) director fluctuations modulate in a slow time scale the residual proton-proton interactions left after averaging by fast molecular motions. The well known square root frequency dependence $\left(T_{1}^{-1}\right)_{\mathrm{DF}} \sim \nu^{-1 / 2}$, obtained for the nematic phase, ${ }^{32,45}$ is modified if the finite dimension of molecules on one side, and the finite size of the nematic domain on the other side, are taken into account. As a result, the square root regime is limited to the frequency range between $\nu_{C_{\max }}^{N}$ on the upper side and $\nu_{C_{\min }}^{N}$ at the lower side. For Larmor frequencies $\nu$ larger than $\nu_{C_{\max }}^{N}$ the dispersion becomes quadratic, whereas it levels-off into a plateau for frequencies smaller than $\nu_{C_{\min }}^{N}$. Such behavior is described by the following equations for the nematic phase: ${ }^{35,46-49}$

$$
\begin{aligned}
\left(\frac{1}{T_{1}}\right)_{\mathrm{DF}_{N}}= & \frac{A_{N}}{\sqrt{\nu}}\left[j_{N}^{(1)}\left(\nu, \nu_{C_{\max }}^{N}, \nu_{C_{\min }}^{N}, \delta\right)\right. \\
& \left.+\frac{1}{\sqrt{2}} j_{N}^{(2)}\left(2 \nu, \nu_{C_{\max }}^{N}, \nu_{C_{\min }}^{N}, \delta\right)\right],
\end{aligned}
$$

where

$j_{N}^{(k)}\left(\nu, \nu_{C_{\max }}^{N}, \nu_{C_{\min }}^{N}, \delta\right)=f_{k 1}(\delta)\left[g_{N}\left(\frac{\nu_{C_{\max }}^{N}}{\nu}\right)-g_{N}\left(\frac{\nu_{C_{\min }}^{N}}{\nu}\right)\right]$

and

$$
\begin{aligned}
g_{N}(a)= & \frac{1}{\pi}(\arctan (\sqrt{2 a}+1)+\arctan (\sqrt{2 a}-1) \\
& \left.-\operatorname{arctanh}\left(\frac{\sqrt{2 a}}{a+1}\right)\right) .
\end{aligned}
$$

The effectiveness of order fluctuations in relaxing the spins is given by the parameter $A_{N}$ which depends on the viscoelastic properties of the liquid crystal, on the square of the orientational order parameter $S$ and on the strength of the proton-proton interaction. $\nu_{C_{\max }}^{N}$ and $\nu_{C_{\min }}^{N}$ are the high and low cut-off frequencies. The angular functions $f_{k 1}(\delta)$ determine the $\left(T_{1}^{-1}\right)_{\mathrm{DF}}(\delta)$ and can be found elsewhere. ${ }^{46,50}$

In the smectic A phase, the director fluctuations are associated with layer undulations. If the compressibility of layers is neglected, such fluctuations preserve the layer spacing and the perpendicular orientation of the molecules with respect to the instantaneous layer normal. They are restricted to wave vectors in the layer plane, i.e., $q_{z}=0$ and yield a linear dependence $T_{1}^{-1} \sim \nu^{-1}$ over a broad frequency range, described by equations ${ }^{18,19,26}$

$$
\begin{aligned}
\left(\frac{1}{T_{1}}\right)_{\mathrm{DF}_{L U}}= & \frac{A_{L U}}{\nu}\left[j_{L U}^{(1)}\left(\nu, \nu_{C_{\max }}^{L U}, \nu_{C_{\min }}^{L U}, \delta\right)\right. \\
& \left.+\frac{1}{2} j_{L U}^{(2)}\left(2 \nu, \nu_{C_{\max }}^{L U}, \nu_{C_{\min }}^{L U}, \delta\right)\right],
\end{aligned}
$$




$$
\begin{aligned}
j_{L U}^{(k)}\left(\nu, \nu_{C_{\max }}^{L U}, \nu_{C_{\min }}^{L U}, \delta\right)= & f_{k 1}(\delta)\left[\frac{2}{\pi} \arctan \left(\frac{\nu_{C_{\max }}^{L U}}{\nu}\right)\right. \\
& \left.-\frac{2}{\pi} \arctan \left(\frac{\nu_{C_{\min }}^{L U}}{\nu}\right)\right],
\end{aligned}
$$

where the same notation is used as in Eq. (4) and LU denotes layer undulations.

As far as we know, there is no theory specific for relaxation induced by director fluctuations in the smectic $\mathrm{C}$ phase. Light scattering studies clearly show that in this phase the fluctuations are not limited to wave vectors in the plane of the layer $\left(q_{z}=0\right)$ but involve also fluctuations of the director, i.e., of the preferred orientation of the long molecular axes, about the layer normal with $q_{z} \neq 0 .{ }^{1}$ In consequence, the smectic $\mathrm{C}$ phase is more turbid in appearance than the smectic A phase. The fluctuations with $q_{z} \neq 0$, which refer to the rotation of the director $\mathbf{n} \equiv\left(n_{x}, n_{y}, n_{z}\right)$ without any distortion of the layers, have the mean square amplitude

$$
\left\langle\left|n_{y}(\mathbf{q})\right|^{2}\right\rangle=\frac{k_{B} T}{\left(B_{1} q_{x}^{2}+B_{2} q_{y}^{2}+B_{3} q_{z}^{2}+2 B_{13} q_{x} q_{z}\right) V},
$$

where $B_{1}, B_{2}, B_{3}$, and $B_{13}$ are the elastic constants of the smectic $C$ phase which are roughly of the same order of magnitude as the nematic elastic constants and $V$ is the sample volume. ${ }^{1}$ The component $n_{y}$ of the director is perpendicular to the plane determined by the layer normal and by the average direction of $\mathbf{n}$. Assuming that the characteristic damping times of the viscoelastic relaxation modes are given by $^{45}$

$$
\tau_{y}(\mathbf{q})=\frac{\eta}{B_{1} q_{x}^{2}+B_{2} q_{y}^{2}+B_{3} q_{z}^{2}+2 B_{13} q_{x} q_{z}},
$$

where $\eta$ is an effective viscosity, we sum up the contributions of all modes by integrating over $q$ from 0 to $\infty$. A dispersion similar to the nematic phase, i.e., $T_{1}^{-1} \propto \nu^{-1 / 2}$, is found. On the other hand, the undulations of the layers in the SmC phase should give a $T_{1}^{-1} \propto \nu^{-1}$ dependence as in the smectic A phase. Neglecting the coupling term between both types of fluctuations, ${ }^{51,52}$ we fit the experimental $T_{1}$ values in the $\mathrm{SmC}$ phase by adding up a contribution of nematiclike fluctuations with strength $A_{N}$ and of smectic fluctuations with strength $A_{L U}$. It should be mentioned that in the chiral smectic $C^{*}$ phase additional modes related to the distortions of the helix should be taken into account. ${ }^{53,54}$

In fitting the total relaxation rate [Eq. (1)] to the experimental data, $A_{N}, A_{L U}$, and the cut-off frequencies can be obtained. However, the high cut-off frequencies $\nu_{C_{\max }}^{N}$ and $\nu_{C_{\max }}^{L U}$ do not have much influence on the model fits since in the frequency region where they constrain the relaxation ( $\mathrm{MHz}$ regime) the DF mechanism is overwhelmed by translational self-diffusion and local molecular reorientations. Therefore, fixed values $\nu_{C_{\max }}^{N}=100 \mathrm{MHz}$ and $\nu_{C_{\max }}^{N}=60 \mathrm{MHz}$ were used in the $\mathrm{N}$ and $\mathrm{SmC}$ fits and $\nu_{C_{\max }}^{L U}=100 \mathrm{MHz}$ in all smectic phases;

(3) molecular rotations/reorientations. Their effect upon nuclear relaxation has been intensively worked out to

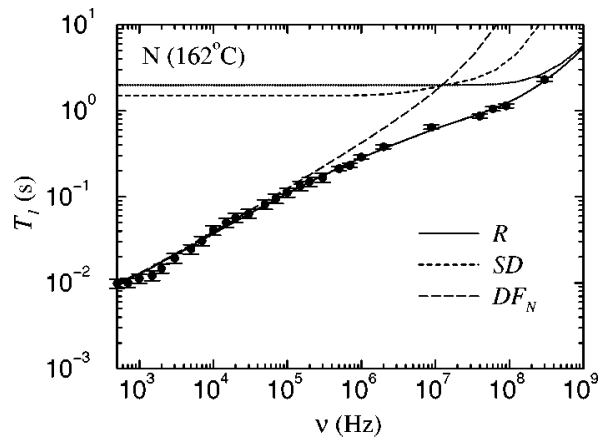

FIG. 5. Frequency dispersion of the proton spin-lattice relaxation time in the nematic phase of $\mathrm{DB}_{8} \mathrm{Cl}$. The solid line represents the fit of Eq. (1) to the experimental data. The contributions of different relaxation mechanisms are indicated. The values of the fitting parameters are given in Table I.

explain the deuterium spin relaxation rate in the $\mathrm{MHz}$ regime in smectic and nematic compounds. ${ }^{16,22,35,55,56}$ Here a rotational diffusion model which assumes that each molecule moves in time in a sequence of small angular steps caused by collisions with surrounding molecules and under the influence of a mean square torque exerted by these molecules is usually used. ${ }^{57-59}$ In the analysis of our data, however, the local molecular rotations and conformational changes give a smaller contribution than in the case of deuterons. They are effective only at the highest frequencies studied. We used, therefore, a simpler model evaluated by Vold et al. ${ }^{60}$ The possible anisotropy of the local molecular long-axisreorientations is neglected,

$$
\begin{aligned}
\left(\frac{1}{T_{1}}\right)_{R}= & \frac{9}{8}\left(\frac{\mu_{0}}{4 \pi}\right)^{2} \gamma^{4} \hbar^{2}\left(J^{(1)}\left(\nu, \delta, A^{(0)}, A^{(1)}, A^{(2)}, \tau_{\perp}, \tau_{\|}, \tau_{\gamma}, S\right)\right. \\
& \left.+J^{(2)}\left(2 \nu, \delta, A^{(0)}, A^{(1)}, A^{(2)}, \tau_{\perp}, \tau_{\|}, \tau_{\gamma}, S\right)\right),
\end{aligned}
$$

where the spectral densities $J^{(k)}$ are given by Vold et al. ${ }^{60}$ $A^{(m)}$ are geometric factors which depend on the intramolecular interproton distances and orientations. $\tau_{\perp}, \tau_{\|}$, and $\tau_{\gamma}$ are the correlation times associated to the molecular reorientations in the director frame $\left(\tau_{\perp}, \tau_{\|}\right)$and to the molecular fast rotations around the long molecular axis in the molecular frame $\left(\tau_{\gamma}\right)$, respectively. $S$ is the orientational order parameter. The geometric factors $A^{(m)}$ are estimated from the intramolecular interproton distances and orientations with respect to a molecular long axes: $A^{(0)} \simeq 4 \times 10^{57} \mathrm{~m}^{-6}, A^{(1)}$ $\simeq 3 \times 10^{57} \mathrm{~m}^{-6}$, and $A^{(2)} \simeq 4 \times 10^{57} \mathrm{~m}^{-6}$. The orientational order parameter $S$ for $\mathrm{DB}_{8} \mathrm{Cl}$ is obtained from proton spectra at different temperatures. Its values are $S=0.46$, $0.63,0.67,0.73,0.73,0.75$, and 0.75 consequently for the $\mathrm{N}$ $\left(T=162{ }^{\circ} \mathrm{C}\right), \mathrm{SmA}_{2}\left(T=145^{\circ} \mathrm{C}\right.$ and $\left.T=135^{\circ} \mathrm{C}\right), \mathrm{SmC}_{2}(T$ $=115^{\circ} \mathrm{C}$ and $\left.T=112^{\circ} \mathrm{C}\right)$, and $\mathrm{SmC}_{?}\left(T=105^{\circ} \mathrm{C}\right.$ and $T$ $=102{ }^{\circ} \mathrm{C}$ ) phases. $\tau_{\perp}, \tau_{\|}$, and $\tau_{\gamma}$ were the fitting parameters of this model.

In the analysis of the angular dependence of $T_{1}^{-1}$ results in the SmA and SmC phases different treatments are required. In the $\mathrm{SmA}_{2}$ phase the director is always parallel to layers' normal and, therefore, $\delta \equiv \Delta$. In the case of the $\mathrm{SmC}_{2}$ and $\mathrm{SmC}_{\text {? }}$ phases it is necessary to take into account a distribution of director orientations, as the sample is rotated in the magnetic field, as described at the end of Sec. II. Besides, 


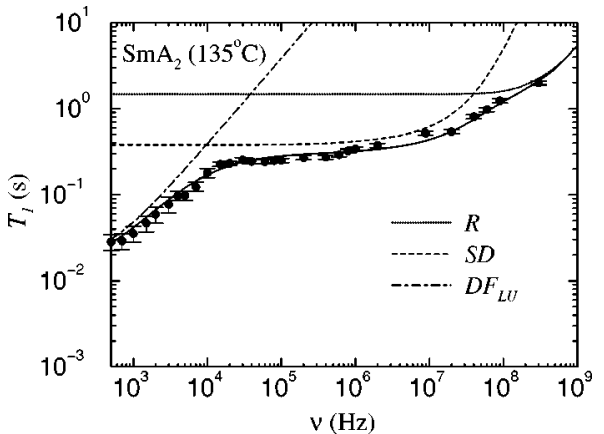

FIG. 6. Frequency dispersion of the proton spin-lattice relaxation time in the $\mathrm{SmA}_{2}$ phase of $\mathrm{DB}_{8} \mathrm{Cl}$. The solid line represents the fit of Eq. (1) to the experimental data. The contributions of different relaxation mechanisms are indicated. The values of the fitting parameters are given in Table I.

in the $\mathrm{SmC}_{\text {? }}$ phase an additional tilt angle $\beta$ of the molecules has to be taken into account in order to reflect the particular structure of this phase, ${ }^{23}$ similar to the antiferroelectric phase formed by chiral molecules. To account for these details in calculating the relaxation rate, the functions $f_{k j}(\delta)$ which appear in Eqs. 4 and 5 [and which are also implicitly involved as spectral densities prefactors in Eqs. (2) and (8)] are replaced by their values averaged over all domains

$$
\overline{f_{k j}(\Delta)}=\sum_{m=1}^{N} f_{k j}\left(\delta_{m}(\Delta)\right) / N
$$

In Eq. (9) the summation is over all domains with different director orientations. In the case of the $\mathrm{SmC}_{2}$ phase, the angle $\delta_{m}(\Delta)$ is obtained by minimizing the free energy in the smectic $\mathrm{C}_{2}$ phase taking into account the elastic and the magnetic energy of the liquid crystal. ${ }^{23}$

\section{ANALYSIS AND DISCUSSION}

In the analysis of the $\mathrm{DB}_{8} \mathrm{Cl}$ experimental data we reduced the number of fitted parameters as much as possible. As a rule six fitting parameters were considered in the optimization process: $\tau_{D \perp}, A\left(A_{N}, A_{L U}\right), \nu_{C_{\min }}\left(\nu_{C_{\min }}^{N}, \nu_{C_{\min }}^{L U}\right)$, $\tau_{\perp}, \tau_{\|}$, and $\tau_{\gamma}$. This might seem to be a large number of parameters, but fortunately different relaxation mechanisms

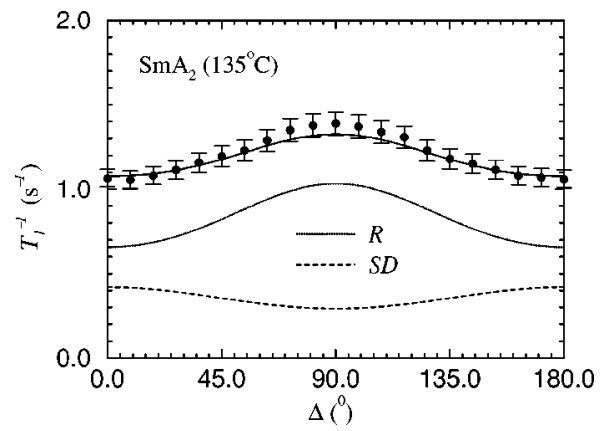

FIG. 7. Angular dependence of the proton spin-lattice relaxation rate $T_{1}^{-1}$ in the $\mathrm{SmA}_{2}$ phase of $\mathrm{DB}_{8} \mathrm{Cl}$ at $\mathrm{T}=135{ }^{\circ} \mathrm{C}$ and $\nu=60 \mathrm{MHz}$. The solid line represents the fit of Eq. (1) to the experimental data. The contributions of $\mathrm{R}$ and SD relaxation mechanisms are indicated (the $D F_{L U}$ contribution is negligible at this frequency). The values of the fitting parameters are identical to those used in the fit presented in Fig. 6.

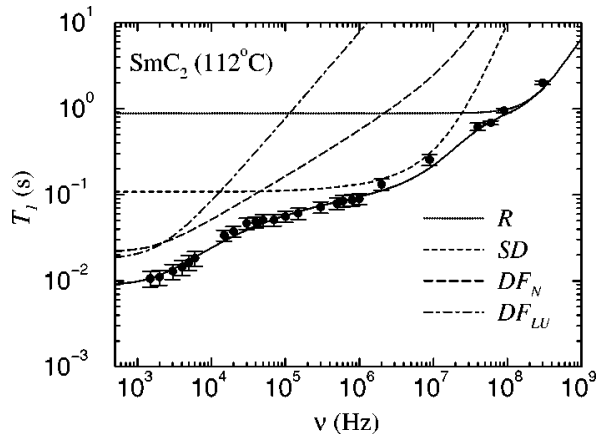

FIG. 8. Frequency dispersion of the proton spin-lattice relaxation time in the $\mathrm{SmC}_{2}$ phase of $\mathrm{DB}_{8} \mathrm{Cl}$. The solid line represents the fit of Eq. (1) to the experimental data. The contributions of different relaxation mechanisms are indicated. The values of the fitting parameters are given in Table I.

act in different frequency ranges. Therefore, the total dispersion curve, which extends over six frequency decades, gives quite reliable values for the above parameters. The results of the fit are further supported by the analysis of angular dependences. The values of parameters, which provide the best fit to the experimental data, are summarized in Table I.

The contributions of different relaxation mechanisms calculated with these parameters and the total relaxation rates are plotted, together with experimental data, in Figs. 5-11.

\section{A. Nematic phase}

As shown in Fig. 5, $T_{1}(\nu)$ in the nematic phase reveals a typical distribution of relaxation mechanisms over the studied frequency range. The DF mechanism dominates the relaxation from $1 \mathrm{kHz}$ to $\sim 10 \mathrm{MHz}$ exhibiting the $T_{1} \sim \nu^{1 / 2}$ dependence. Its "strength" $A_{N}=2600 \mathrm{~s}^{-3 / 2}$ at $162^{\circ} \mathrm{C}$ is slightly larger than the values obtained for similar compounds. ${ }^{18,19}$ Translational self-diffusion and rotations/ reorientations prevail as relaxation mechanisms in the high frequency range. The self-diffusion coefficient $D_{\perp}$, estimated from $D_{\perp}=\left\langle r_{\perp}^{2}\right\rangle / 4 \tau_{D \perp}$, amounts to $9 \times 10^{-11} \mathrm{~m}^{2} / \mathrm{s}$. The measurements of the angular dependence of $T_{1}^{-1}$ in the nematic phase could not be performed without special equipment or a more elaborated experimental technique, as the director adjusts to the magnetic field in a very short time..$^{19,20}$

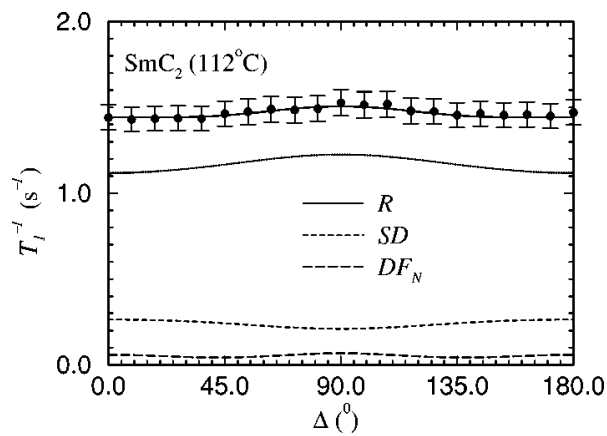

FIG. 9. Angular dependence of the proton spin-lattice relaxation rate $T_{1}^{-1}$ in the $\mathrm{SmC}_{2}$ phase of $\mathrm{DB}_{8} \mathrm{Cl}$ at $\mathrm{T}=112{ }^{\circ} \mathrm{C}$ and $\nu=60 \mathrm{MHz}$. The solid line represents the fit of Eq. (1) to the experimental data. The contributions of different relaxation mechanisms effective at this frequency are indicated (the $\mathrm{DF}_{L U}$ contribution is negligible at $60 \mathrm{MHz}$ ). The values of the fitting parameters are identical to those used in the fit presented in Fig. 8. 


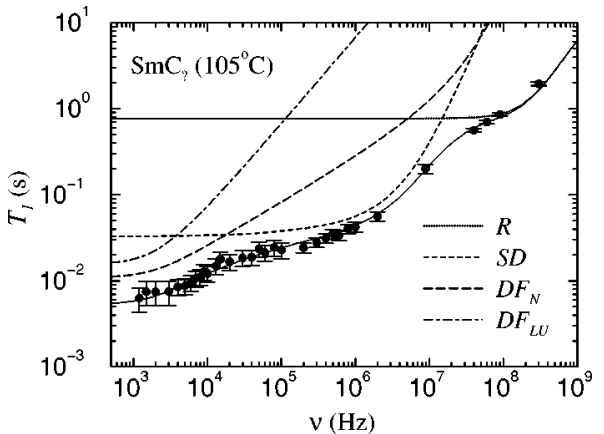

FIG. 10. Frequency dispersion of the proton spin-lattice relaxation time in the $\mathrm{SmC}_{\text {? }}$ phase of $\mathrm{DB}_{8} \mathrm{Cl}$. The solid line represents the fit of Eq. (1) to the experimental data. The contributions of different relaxation mechanisms are indicated. The values of the fitting parameters are given in Table I.

\section{B. Bilayer smectic A phase $\left(\mathrm{SmA}_{2}\right)$}

The $T_{1}$ versus $\nu$ in the $\mathrm{SmA}_{2}$ phase is presented in Fig. 6 and $T_{1}^{-1}$ versus $\Delta$ in Fig. 7. The experimental data clearly indicate that a relaxation mechanism yielding $T_{1} \sim \nu^{1}$ frequency dependence is effective below $10^{4} \mathrm{~Hz}$. It is also indirectly responsible for the "shoulder" in the dispersion curve, which is not observed in the nematic phase. This mechanism can be-in view of its specific dispersionassigned most probably to layer undulations, though the impact of layers' compressibility on cut-off frequencies has not yet been experimentally clarified. ${ }^{61}$ In our case the low cutoff frequency could not be precisely determined as no true low-frequency plateau was detected in $T_{1}(\nu)$. The value of $\nu_{C_{\text {min }}}^{L U}$ presented in Table I is merely indicative.

The relaxation in the intermediate frequency range (10 $\mathrm{kHz}-20 \mathrm{MHz}$ ) is dominated by translational self-diffusion, which is slower that in the nematic phase. The estimated diffusion coefficient is $D_{\perp}=5 \times 10^{-11} \mathrm{~m}^{2} / \mathrm{s}$. Rotations/ reorientations are important only in the high-frequency regime $(>20 \mathrm{MHz})$.

All parameters obtained in the fit of dispersion curves can be here additionally tested by the angular dependence of the relaxation rate. Parameters listed in Table I were used to plot the solid line in Fig. 7. Obviously it matches well the experimental data. The observed maximum at $\Delta=90^{\circ}$ is due mainly to the contribution of the rotation/reorientations mechanism.

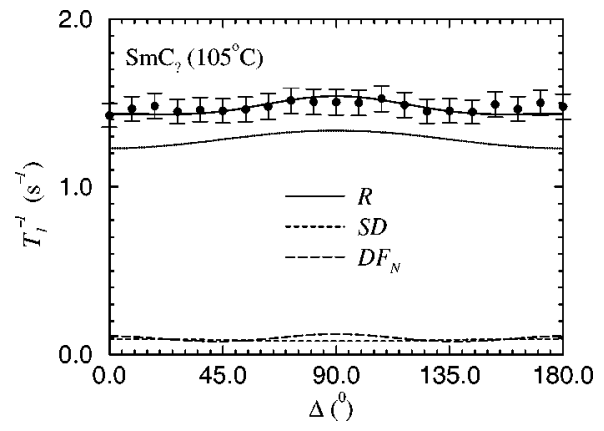

FIG. 11. Angular dependence of the proton spin-lattice relaxation rate $T_{1}^{-1}$ in the $\mathrm{SmC}_{\text {? }}$ phase of $\mathrm{DB}_{8} \mathrm{Cl}$ at $\mathrm{T}=105{ }^{\circ} \mathrm{C}$ and $\nu=60 \mathrm{MHz}$. The solid line represents the fit of Eq. (1) to the experimental data. The contributions of different relaxation mechanisms effective at this frequency are indicated (the $\mathrm{DF}_{L U}$ contribution is negligible at $60 \mathrm{MHz}$ ). The values of the fitting parameters are identical to those used in the fit presented in Fig. 10.

\section{Bilayer tilted smectic phase $\left(\mathrm{SmC}_{2}\right)$}

Measurements of the frequency dependence of $T_{1}^{-1}$ in the bilayer $\mathrm{SmC}_{2}$ phase at $T=112^{\circ} \mathrm{C}$ are presented in Fig. 8 . The contributions of rotations/reorientations and particularly of self-diffusion to $T_{1}^{-1}$ have obviously increased compared to the nematic and smectic A phases. This is due to an increase of parameters $\tau_{\perp}, \tau_{\|}, \tau_{\gamma}$ for rotations and of $\tau_{D \perp}$ for translational diffusion with decreasing temperature, as expected for thermally activated motions. The angular dependence of $T_{1}^{-1}$ at $60 \mathrm{MHz}$, presented in Fig. 9, is much less pronounced than in the $\mathrm{SmA}_{2}$ phase and hardly exceeds the experimental error. This is due to a relatively smaller contribution of local rotations to the total relaxation rate at $60 \mathrm{MHz}$. The flat $T_{1}^{-1}(\Delta)$ curve is well explained by the compensating effect of the self-diffusion and rotations/ reorientations relaxation mechanisms. Since both the frequency and the angular dependences of $T_{1}^{-1}$ in the $\mathrm{MHz}$ regime can be described by the same values of fitted parameters, we are reasonably sure that the interpretation is correct in spite of a large number of parameters involved.

A particularly interesting point in our study is the proton relaxation in the $\mathrm{kHz}$ regime. According to earlier studies of the smectic $\mathrm{C}$ phase, director fluctuations should be dominant in this frequency range, but the frequency dependence of their contribution to $T_{1}^{-1}$ has not been well establi-

TABLE I. Parameters describing molecular dynamics in $\mathrm{DB}_{8} \mathrm{Cl}$ as obtained from the fit of theoretical expressions (described in the text) to the measured $T_{1}^{-1}$ vs $\nu^{-1}$ and $T_{1}^{-1}$ vs $\Delta$ dependences.

\begin{tabular}{|c|c|c|c|c|c|c|c|c|}
\hline \multirow{2}{*}{\multicolumn{2}{|c|}{$\begin{array}{l}\text { Phase } \\
T\left({ }^{\circ} \mathrm{C}\right)\end{array}$}} & \multirow{3}{*}{$\begin{array}{c}\mathrm{N} \\
162 \\
0.68\end{array}$} & \multicolumn{2}{|c|}{$\mathrm{SmA}_{2}$} & \multicolumn{2}{|c|}{$\mathrm{SmC}_{2}$} & \multicolumn{2}{|c|}{$\mathrm{SmC}_{?}$} \\
\hline & & & \multirow{2}{*}{$\begin{array}{l}145 \\
1.2\end{array}$} & \multirow{2}{*}{$\begin{array}{l}135 \\
2.1\end{array}$} & \multirow{2}{*}{$\begin{array}{l}115 \\
4.3\end{array}$} & \multirow{2}{*}{$\frac{112}{6.3}$} & \multirow{2}{*}{$\begin{array}{c}105 \\
19\end{array}$} & \multirow{2}{*}{$\frac{102}{27}$} \\
\hline SD & $\tau_{D \perp}\left(10^{-9} \mathrm{~s}\right)$ & & & & & & & \\
\hline \multirow{4}{*}{ DF } & $\nu_{C_{\text {min }}}^{N}\left(10^{3} \mathrm{~Hz}\right)$ & $\sim 0.03$ & $\ldots$ & $\ldots$ & 1.5 & 1.5 & 1.5 & 1.6 \\
\hline & $A_{N}\left(10^{3} \mathrm{~s}^{-3 / 2}\right)$ & 2.6 & $\ldots$ & $\ldots$ & 1.9 & 2.0 & 4.0 & 6.0 \\
\hline & $\nu_{C}^{L U}\left(10^{3} \mathrm{~Hz}\right)$ & $\ldots$ & 0.36 & 0.32 & 1.5 & 1.5 & 1.5 & 1.5 \\
\hline & $A_{L U}\left(10^{4} \mathrm{~s}^{-2}\right)$ & $\ldots$ & 2.2 & 2.6 & 13 & 13 & 15 & 16 \\
\hline \multirow{3}{*}{$\mathrm{R}$} & $\tau_{\perp}\left(10^{-10} \mathrm{~s}\right)$ & 0.73 & 0.78 & 1.0 & 2.3 & 3.0 & 3.4 & 3.4 \\
\hline & $\tau_{\|}\left(10^{-11} \mathrm{~s}\right)$ & 0.10 & 0.18 & 0.28 & 0.97 & 1.4 & 1.7 & 1.8 \\
\hline & $\tau_{\gamma}\left(10^{-12} \mathrm{~s}\right)$ & 0.63 & 1.1 & 1.7 & 8.2 & 11 & 17 & 18 \\
\hline
\end{tabular}


shed. ${ }^{21,27,34}$ We find that $T_{1}^{-1}$ of $\mathrm{DB}_{8} \mathrm{Cl}$ in the $\mathrm{kHz}$ range cannot be fitted either with $T_{1}^{-1} \sim \nu^{-1 / 2}$ nor with $T_{1}^{-1} \sim \nu^{-1}$ dispersion alone. Only a superposition of both dispersion "laws" is capable of explaining the experimental data (Fig. 8). This fact is not surprising if we take into account that in the tilted smectic phase the director can reorient about the layer normal without disturbing the layer thickness. This motion, described by Eqs. (6) and (7), leads to a relaxation rate proportional to $\nu^{-1 / 2}$ in the range between the low and the high cut-off frequencies, as shown in Sec. III-2. The fit, presented in Fig. 8, shows that the contribution of in-layer fluctuations of the director exceeds that of layer undulations in the whole frequency range studied. Its effectiveness is given by the parameter $A_{N}=2.0 \times 10^{3} \mathrm{~s}^{-3 / 2}$ (see Table I), which is only slightly smaller than in the nematic phase. This might seem unusual since the director in the smectic C is "free" to rotate only in the plane of the layer, whereas the out-of-plane fluctuations are much more restricted. However, a similar value of the parameters $A_{N}$ for both mesophases may result from increased viscosity and orientational order parameter in the $\mathrm{SmC}_{2}$ phase. ${ }^{62}$ The constant describing the effectiveness of layer undulations, $A_{L U}$, is roughly 5 times larger in the $\mathrm{SmC}_{2}$ phase than in the $\mathrm{SmA}$ phase because of increased positional, i.e., smectic ordering. The values of the cut-off frequencies, $\nu_{C_{\min }}^{N}$ and $\nu_{C_{\min }}^{L U}$, given in Table I, are only indicative. They should be larger than in the nematic or smectic A phase, respectively, as a low-frequency plateau in the dispersion curve between $10^{3}$ and $10^{4} \mathrm{~Hz}$ is here clearly observed. But the data do not allow to decide whether this is caused by of the onset of the frequency cutoff or by the transition from Zeeman into the dipolar spin "reservoir" which takes place in the same frequency range.

\section{Anticliniclike smectic C phase $\left(\mathrm{SmC}_{\text {? }}\right)$}

Frequency and angular dependences of the proton relaxation in the $\mathrm{SmC}_{\text {? }}$ phase are presented in Figs. 10 and 11, together with the best fit of Eq. (1) to the experimental data. The measurements are explained basically by the same relaxation mechanisms as in the $\mathrm{SmC}_{2}$ phase, but there are some important differences. Translational self-diffusion is considerably slower in the low-temperature tilted phase. Consequently its importance in the spin relaxation process is increased. Self-diffusion is here the dominating relaxation mechanism in the whole frequency range between $2 \times 10^{4}$ $\mathrm{Hz}$ and $2 \times 10^{7} \mathrm{~Hz}$, i.e., over three frequency decades. The in-plane correlation time for translational diffusion, $\tau_{D \perp}$, exhibits an abrupt increase by a factor of 3 at the transition from $\mathrm{SmC}_{2}$ to the $\mathrm{SmC}_{\text {? }}$ phase. This indicates that in the $\mathrm{SmC}_{\text {? }}$ phase the diffusion in the bilayer is hindered by the formation of molecular pairs with anticlinic arrangement. It should be also stressed that the best fit was obtained in the limit $D_{\|}^{0} / D_{\perp}^{0} \rightarrow 0$. Obviously a strong slowing-down of molecular exchange between the layers takes place supporting again the conjecture of anticlinic molecular ordering. The contribution of in-plane director fluctuations to $T_{1}^{-1}$ in $\mathrm{DB}_{8} \mathrm{Cl}$ is increased as well, most probably due to the increase in the viscosity coefficient. The determination of the contribution of layer undulations is less reliable as there is no frequency region where this contribution would dominate. Therefore, the magnitude of $\left(T_{1}\right)_{D F_{L U}}$ was kept here at values close to the ones obtained in the $\mathrm{SmC}_{2}$ phase. It was not possible to perform a more detailed study of the $T_{1}$ dispersion at low frequencies since the $T_{1}$ values in the $\mathrm{SmC}_{\text {? }}$ phase are so small that they are close to the fast field-cycling spectrometer's experimental limit.

\section{CONCLUSIONS}

In conclusion we may say that a comprehensive study of proton spin-lattice relaxation in the nematic and bilayer smectic phases of $\mathrm{DB}_{8} \mathrm{Cl}$ has been performed. Though the same relaxation mechanisms are basically present in all mesophases, their parameters obtained in the fit reveal the specific character of each mesophase. The correlation times associated with local molecular reorientations, which are the most effective relaxation mechanism in the high-frequency regime, increase with decreasing temperature in the usual way, characteristic of thermally activated motions. On the other hand, translational self-diffusion dominating the proton relaxation at intermediate frequencies, shows an abrupt increase in the intra-layer correlation time at the transition from the $\mathrm{SmC}_{2}$ into $\mathrm{SmC}_{\text {? }}$ phase. Besides, the best fit in this phase is obtained in the limit $D_{\|}^{0} / D_{\perp}^{0} \rightarrow 0$ indicating a considerable decrease of out-of-layer molecular diffusion as well. These facts-slowing-down of intra-layer and particularly of out-of-layer molecular diffusion-indirectly support the anticlinic arrangement of molecular pairs in the $\mathrm{SmC}_{\text {? }}$ phase. A similar effect was observed in antiferroelectric liquid crystals, where the anticlinic molecular ordering diminishes the diffusion between layers by two orders of magnitude compared to ferroelectric liquid crystals with uniform short range tilt. ${ }^{63}$

Director fluctuations dominate the relaxation in the lowest frequency regime under study. In the nematic phase the usual square root dispersion profile is observed. In the $\mathrm{SmA}_{2}$ phase a linear frequency dependence of $\left(T_{1}^{-1}\right)_{\mathrm{DF}}$ explains the experimental data. It is ascribed to the undulations of the layers. In contrast to former conjectures, we find that the $T_{1}^{-1}$ low-frequency dispersion in the $\mathrm{SmC}_{2}$ and $\mathrm{SmC}_{\text {? }}$ phases can be explained only if - in addition to layer undulationsfluctuations of the molecular director about the layer normal, which occur without distortion of the layers, are taken into account. Both types of fluctuations are well-known from optical studies but have not been identified by NMR so far. ${ }^{62}$ The calculated contribution of the in-plane director fluctuations in the tilted smectic phases yields a dispersion $\left(T_{1}^{-1}\right)_{\mathrm{DF}} \sim \nu^{-1 / 2}$, similar to nematic fluctuations. However, the nematiclike $T_{1}$ dispersion in the $\mathrm{SmC}_{2}$ and $\mathrm{SmC}_{\text {? }}$ phases is not a matter of nematic domains in the smectic phase or a pretransitional effect but an intrinsic property of tilted smectic phases.

\section{ACKNOWLEDGMENTS}

The authors wish to thank Fundação para a Ciência e Tecnologia (FCT) through projects PBIC/C/CTM/1935/95 and PRAXIS XXI 3/3.1/MMA/1769/95. One of the authors 
(A.C.) thanks FCT for Grant No. PRAXIS XXI BD/2900/94 and M.V. acknowledges the Ministry of Science and Technology of Slovenia, Grant No. P1-024. We also thank Dr. Gabriel Feio for the experimental help with the Bruker MSL spectrometer, and Dr. Benoit Heinrich for scientific discussions.

${ }^{1}$ S. Chandrasekhar, Liquid Crystals (Cambridge University Press, Cambridge, 1992).

${ }^{2}$ J. Goodby, in Handbook of Liquid Crystals, edited by D. Demus, J. W. Goodby, G. W. Gray, H. W. Spiess, and V. Vill (Wiley-VCH, New York, 1998).

${ }^{3}$ A. Leadbetter, J. L. Durrant, and M. Rugman, Mol. Cryst. Liq. Cryst. 34, 231 (1977).

${ }^{4}$ P. Barois, J. Prost, and T. C. Lubensky, J. Physique 46, 391 (1985).

${ }^{5}$ J. Prost and P. Barois, J. de Chim. Phys. 80, 66 (1983).

${ }^{6}$ H. Nguyen, J. de Chim. Phys. 80, 83 (1983).

${ }^{7}$ F. Hardouin and A. M. Levelut, J. Physique 41, 41 (1980).

${ }^{8}$ F. Hardouin, A. M. Levelut, and G. Sigaud, J. Physique 42, 71 (1981).

${ }^{9}$ F. Hardouin, H. Nguyen, and A. M. Levelut, J. Phys. (France) Lett. 43, L1779 (1982).

${ }^{10}$ N. Nuu Tinh, F. Hardouin, and C. Destrade, J. Physique 43, 1127 (1982).

${ }^{11}$ F. Hardouin, A. Levelut, G. Sigaud, M. Achard, N. Tinh, and H. Gasparoux, Symmetries and Broken Symmetries in Condensed Matter Physics, N. Boccara (IDSET-Paris) (1981).

${ }^{12}$ V. Raja, R. Shashidhar, B. Ratna, G. Heppke, and C. Bahr, Phys. Rev. A 37, 303 (1988)

${ }^{13}$ G. Sigaud, F. Hardouin, M. F. Achard, and A. M. Levelut, J. Physique 42, 107 (1981).

${ }^{14}$ A. Levelut, R. Tarento, F. Hardouin, M. Achard, and G. Sigaud, Phys. Rev. A 24, 2180 (1981).

${ }^{15}$ Y. H. Jeong, K. J. Stine, C. W. Garland, and H. Nguyen, Phys. Rev. A 37, 3465 (1988).

${ }^{16}$ R. Y. Dong, Nuclear Magnetic Resonance of Liquid Crystals (SpringerVerlag, Berlin, 1994).

${ }^{17}$ A. Ribeiro, P. Sebastião, and M. Vilfan, Liq. Cryst. 3, 937 (1988).

${ }^{18}$ P. J. Sebastião, A. C. Ribeiro, H. Nguyen, and F. Noack, Z. Naturforsch., A: Phys. Sci. A48, 851 (1993).

${ }^{19}$ P. J. Sebastião, A. C. Ribeiro, and F. Noack, J. Phys. II 5, 1707 (1995).

${ }^{20}$ S. Struppe and F. Noack, Liq. Cryst. 20, 595 (1996).

${ }^{21}$ R. Acosta and D. Pusiol, Phys. Rev. E 60, 1808 (1999).

${ }^{22}$ R. Y. Dong, Phys. Rev. E 60, 5631 (1999).

${ }^{23}$ A. Carvalho, P. Sebastião, A. Ferraz, A. Ribeiro, and H. Nguyen, Eur. Phys. J. E 2, 351 (2000).

${ }^{24}$ L. Benguigui and F. Hardouin, J. Phys. (France) Lett. 45, L179 (1984).

${ }^{25}$ Y. Galerne and L. Liebert, Phys. Rev. Lett. 64, 906 (1990).

${ }^{26}$ R. Blinc, M. Luzar, M. Vilfan, and M. Burgar, J. Chem. Phys. 63, 3445 (1975).

${ }^{27}$ R. Blinc, M. Vilfan, M. Luzar, J. Seliger, and V. Zagar, J. Chem. Phys. 68, 303 (1978).

${ }^{28}$ F. Noack, M. Notter, and W. Weiß, Liq. Cryst. 3, 907 (1988).

${ }^{29}$ F. Noack and K. H. Schweikert, in The Molecular Dynamics of Liquid Crystals, edited by G. Luckhurst and C. A. Veracini (Kluwer Academic, Netherlands, 1994).

${ }^{30}$ W. Wölfel, F. Noack, and M. Stohrer, Z. Naturforsch., A: Phys. Sci. 30, 437 (1975).
${ }^{31}$ F. Noack, Prog. Nucl. Magn. Reson. Spectrosc. 18, 171 (1986).

${ }^{32}$ P. Pincus, Solid State Commun. 7, 415 (1969).

${ }^{33}$ T. Mugele, V. Graf, W. Wölfel, and F. Noack, Z. Naturforsch., A: Phys. Sci. 35, 924 (1980)

${ }^{34}$ C. Cruz, J. L. Figueirinhas, P. J. Sebastião, A. C. Ribeiro, F. Noack, H. T. Nguyen, B. Heinrich, and D. Guillon, Z. Naturforsch., A: Phys. Sci. 51, 155 (1996).

${ }^{35}$ R. Vold and R. Vold, in The Molecular Dynamics of Liquid Crystals, edited by G. Luckhurst and C. A. Veracini (Kluwer Academic, Netherlands, 1994), p. 2.

${ }^{36}$ A. Carvalho, P. J. Sebastião, and H. T. Nguyen, Mol. Cryst. Liq. Cryst. 331, 89 (1999).

${ }^{37}$ E. Rommel, K. Mischker, G. Osswald, K. H. Schweikert, and F. Noack, J. Magn. Reson. (1969-1992) 70, 219 (1986).

${ }^{38}$ R. A. Wise, D. H. Smith, and J. W. Doane, Phys. Rev. A 7 (4), 1366 (1973).

${ }^{39}$ K. H. Schweikert and F. Noack, Z. Naturforsch., A: Phys. Sci. 44, 597 (1989).

${ }^{40}$ S. Žumer and M. Vilfan, Phys. Rev. A 17, 424 (1978).

${ }^{41}$ M. Vilfan and S. Žumer, Phys. Rev. A 21, 672 (1980).

${ }^{42}$ R. Blinc, M. Burgar, M. Luzar, J. Piršs, I. Zupančič, and S. Žumer, Phys. Rev. Lett. 33, 1192 (1974).

${ }^{43} \mathrm{~B}$. Heinrich, Etude de la structure et propriétés mésomorphes de séries homologues et de mélanges de composés smectogènes, Ph.D. thesis, Université Louis Pasteur de Strasbourg (1993).

${ }^{44}$ G. J. Krüger, Phys. Rep. 82, 229 (1982).

${ }^{45} \mathrm{P}$. de Gennes and J. Prost, The Physics of Liquid Crystals (Oxford University Press, 2nd ed., 1993), section 5.2.5.

${ }^{46}$ J. W. Doane, C. E. Tarr, and M. A. Nickerson, Phys. Rev. Lett. 33, 620 (1974).

${ }^{47}$ R. Blinc, M. Vilfan, and V. Rutar, Solid State Commun. 17, 171 (1975).

${ }^{48}$ I. Zupančič, V. Žagar, R. Rožmarin, I. Lesvtik, F. Kogovšik, and R. Blinc, Solid State Commun. 18, 1591 (1976).

${ }^{49}$ J. H. Freed, J. Chem. Phys. 66, 4183 (1977).

${ }^{50}$ P. J. Sebastião, M. H. Godinho, A. C. Ribeiro, D. Guillon, and M. Vilfan, Liq. Cryst. 11, 621 (1992).

${ }^{51}$ R. Blinc, M. Vilfan, and J. Seliger, Bull. Magn. Reson. 5, 51 (1983).

${ }^{52}$ B. Zalar, O. Lavrentovich, H. Zeng, and D. Finotello, Phys. Rev. E 62, 2252 (2000).

${ }^{53}$ I. Muševič, A. Rastegar, M. Čepič, B. Žekš, M. Čopič, D. Moro, and G. Heppke, Phys. Rev. Lett. 77, 1769 (1996).

${ }^{54}$ A. Ferraz, A. C. Ribeiro, and H. T. Nguyen, Mol. Cryst. Liq. Cryst. 331, 67 (1999)

${ }^{55}$ R. Y. Dong and M. Cheng, J. Chem. Phys. 113, 3466 (2000).

${ }^{56}$ R. Y. Dong, A. Carvalho, P. J. Sebastião, and H. T. Nguyen, Phys. Rev. E 62, 3679 (2000).

${ }^{57}$ P. Nordio and P. Busolin, J. Chem. Phys. 55, 5485 (1971).

${ }^{58}$ A. Ferrarini, G. J. Moro, and P. L. Nordio, Liq. Cryst. 8, 593 (1990).

${ }^{59}$ R. Y. Dong, Phys. Rev. A 43, 4310 (1991).

${ }^{60}$ R. R. Vold and R. G. Vold, J. Chem. Phys. 88, 1443 (1988).

${ }^{61}$ M. Vilfan, M. Kogoj, and R. Blinc, J. Chem. Phys. 86, 1055 (1987).

${ }^{62}$ Y. Galerne, J. Martinand, G. Durand, and M. Veyssie, Phys. Rev. Lett. 29, 562 (1972).

${ }^{63}$ B. Zalar, A. Gregorovič, and R. Blinc, Phys. Rev. E 62, R37 (2000). 